\title{
Effects of submaximal cycling at different exercise intensities on maximal isometric force output of the non-exercised elbow flexor muscles
}

\author{
R Matsuura ${ }^{1,2}$, K Hirakoba $^{3}$, K Takahashi $^{4}$ \\ ${ }^{1}$ Living and Health Sciences Education, Specialized Subject Fields of Education, Graduate School of Education, \\ Joetsu University of Education, Joetsu, Japan \\ ${ }^{2}$ The Joint Graduate School in Science of School Education, Hyogo University of Teacher Education, Kato, Japan \\ ${ }^{3}$ Department of Biological Functions Engineering, Graduate School of Life Science and Systems Engineering, \\ Kyushu Institute of Technology, Kitakyushu, Japan \\ ${ }^{4}$ Faculty of Liberal Studies, National Institute of Technology, Kumamoto College, Koshi, Japan
}

Received: October 2, 2017

Accepted: May 23, 2018

The purpose of this study was to examine the effects of submaximal cycling at different exercise intensities on maximal isometric force output of the non-exercised elbow flexor muscles after the cycling. A total of 8 healthy young men performed multiple maximal voluntary contractions by the right elbow flexion before, immediately after, 5 min after, and 10 min after a 6-min submaximal cycling at ventilatory threshold (LI), $70 \% \mathrm{VO}_{2 \text { peak }}(\mathrm{MI})$, and $80 \%$ $\mathrm{VO}_{2 \text { peak }}(\mathrm{HI})$ with both arms relaxed in the air. Force and surface electromyogram (EMG) of the right biceps brachii muscle during the multiple MVCs, blood lactate concentration ([La]), cardiorespiratory responses, and sensations of fatigue for legs (SEF-L) were measured before, immediately after, $5 \mathrm{~min}$ after, and $10 \mathrm{~min}$ after the submaximal cycling with the three different exercise intensities. Immediately after the submaximal cycling, [La], cardiorespiratory responses, and SEF-L were enhanced in proportion to an increase in exercise intensity of the cycling. Changes in force and EMG activity during the multiple MVCs were not significantly different across the three conditions. The findings imply that group III/IV muscle afferent feedback after the submaximal cycling does not determine the magnitude of MVC force loss of the non-exercised upper limb muscles.

Keywords: muscle fatigue, afferent feedback, central nervous system, blood lactate concentration, electromyogram

\section{Introduction}

Recently, it has been shown that fatiguing muscle contraction results in a reduction of the time to task failure or maximal voluntary contraction (MVC) force in a muscle group is not involved in the exercise $(3,10,19)$. This aspect of muscle fatigue is termed as non-local muscle fatigue (NLMF). Although it is unclear what exactly leads to NLMF, some investigators have proposed that feedback by group III/IV muscle afferents originating in the exercised muscle may contribute to the NLMF $(12-14,20)$. Most of the investigators utilized postexercise circulatory occlusion, which maintains the discharge of group III/V muscle afferents (12-14), and an administration of intrathecal fentanyl, which partially attenuates group III/IV afferent feedback from the exercised muscle (20), to elucidate the effects of group III/IV muscle

Corresponding author: Ryouta Matsuura

Living and Health Sciences Education, Specialized Subject Fields of Education, Graduate School of Education, Joetsu University of Education, 1 Yamayashiki-machi, Joetsu 943-8512, Japan

Phone/Fax: +81 25521 3569; E-mail: matsuura@juen.ac.jp 
afferents in the exercised muscle on NLMF. However, the muscle afferents activated by postexercise circulatory occlusion are different from a subset of group III/IV muscle afferents activated during normal, freely perfused voluntary exercise $(6,10)$. This means that the previous studies involving postexercise circulatory occlusion could not demonstrate whether changes in group III/IV afferent feedback induced by voluntary exercise contribute to NLMF. Furthermore, the pharmacological study (20) that used intrathecal fentanyl did not investigate whether the degree of NLMF is dependent on the magnitude of the effect of administration of lumbar intrathecal fentanyl on group III/IV afferent feedback; however, the study found that attenuation of group III/IV afferent feedback by intrathecal fentanyl prevented the induction of NLMF. Therefore, the effects of the magnitude of exercise-induced changes in the group III/IV afferent feedback on NLMF have yet to be fully elucidated. It is widely known that exercise intensity determines the muscle metabolic demand during constant-duration exercise, resulting in differences in metabolic milieu (i.e., group III/IV afferent feedback). As a result, group III/IV afferent feedback determines the degree of NLMF, and the intensity of constant-duration exercise may be an influencing factor.

The aim of this study was to determine the effects of three different exercise intensities of a constant-duration submaximal cycling on MVC force exerted by the non-exercised elbow flexor muscles after the cycling. We hypothesized that MVC force of the elbow flexor muscles after a high-intensity (HI) cycling would be lower than that after a low-intensity (LI) cycling.

\section{Materials and Methods}

A total of 8 healthy males (mean \pm SD: $24.0 \pm 4.1$ years; height: $173.6 \pm 3.6 \mathrm{~cm}$; and mass: $62.6 \pm 5.0 \mathrm{~kg}$ ) participated in this study. All participants were right-handed. All subjects gave written informed consent, and the experimental procedures were conducted in accordance with the Declaration of Helsinki. The Local Ethics Committee approved the study. The subjects were requested to abstain from strenuous physical activity, alcohol, and caffeine for $24 \mathrm{~h}$ prior to each experimental session. Each subject participated in a preliminary session involving an incremental cycling test and MVC familiarization tests of elbow flexor muscles and three submaximal cycling sessions, which were conducted on separate days. The time interval between two consecutive sessions was at least $48 \mathrm{~h}$. All cycling tests were performed on a cycle ergometer (Monark 828E, Monark Exercise AB, Sweden). Throughout all cycling exercises, the subjects did not grip the ergometer's handle (i.e., both arms were relaxed in the air).

On the first day of the session, the subjects' anthropometric variables were measured and each participant completed an incremental cycling test on the cycle ergometer to volitional exhaustion in order to determine peak oxygen uptake $\left(\mathrm{VO}_{2 \text { peak }}\right)$ and ventilatory threshold (VT). Subjects began cycling at $60 \mathrm{~W}$ for 2 min and then the power output was increased in incremental steps of $30 \mathrm{~W}$ for every 2 min until exhaustion, which was defined as a drop in pedal frequency below $5 \mathrm{rpm}$ of target cadence $(60 \mathrm{rpm})$ for $5 \mathrm{~s}$ despite verbal encouragement. Maximum workload ( $\mathrm{W}_{\max }: 188 \pm 19 \mathrm{~W}$ ) was calculated as follows:

$$
\mathrm{W}_{\max }=\mathrm{W}_{\text {completed }}+[(t / 120) \times 30],
$$

where $\mathrm{W}_{\text {completed }}$ is the power output (W) in the final completed stage and $t$ is the time (s) spent in the final non-completed stage (11). $\mathrm{VO}_{2 \text { peak }}(2,804 \pm 301 \mathrm{ml} / \mathrm{min}$, $45.1 \pm 6.2 \mathrm{ml} / \mathrm{min} / \mathrm{kg}$ ) was determined by averaging breath-by-breath $\mathrm{VO}_{2}$ over a $15-\mathrm{s}$ 


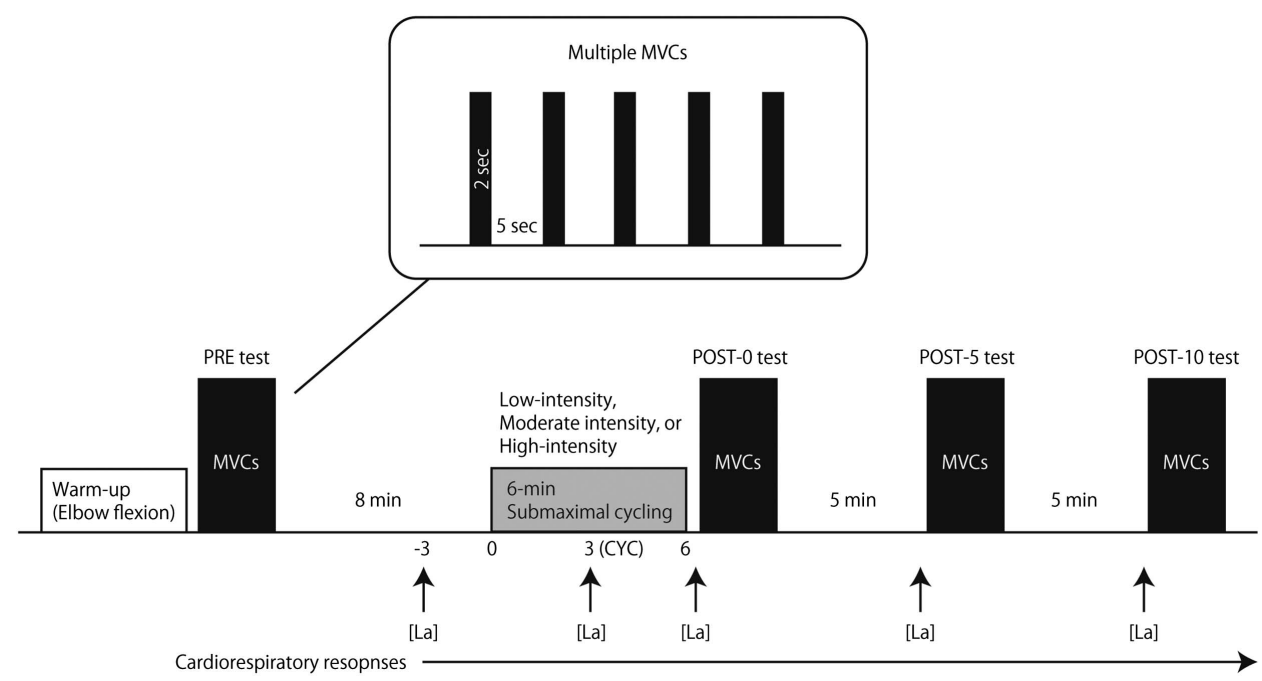

Fig. 1. Schematic presentation of the experimental protocol. The subject was asked to perform multiple maximal voluntary contractions (MVCs) tests with right elbow flexion before (PRE), immediately after (POST-0), 5min after (POST-5), and 10 min after (POST-10) a 6-min submaximal cycling task at low, moderate, or high intensities.

[La]: blood lactate concentration; CYC: time point at $3 \mathrm{~min}$ during the cycling

interval. VT was determined based on the V-slope method (5). Subjects performed MVC tests before and immediately after the incremental cycling test as described below.

On separate days, each subject performed three submaximal cycling sessions (Fig. 1). In each session, subjects performed submaximal constant-load cycling for 6 min using the cycle ergometer. The three experimental conditions were (i) LI cycling (VT: $45.5 \% \pm 3.9 \%$ of $\dot{\mathrm{VO}}{ }_{2 \text { peak }}$, LI condition); (ii) moderate-intensity (MI) cycling ( $70 \%$ of $\dot{\mathrm{VO}}_{2 \text { peak }}, \mathrm{MI}$ condition); and (iii) $\mathrm{HI}$ cycling ( $80 \%$ of $\mathrm{VO}_{2 \text { peak }}$, $\mathrm{HI}$ condition). The order of the conditions was randomly assigned. During the sessions, the MVC test was initially conducted (PRE test) while subjects were seated on the cycle ergometer, which enabled subjects to perform the MVC tests as soon as possible $(<5 \mathrm{~s})$ after the submaximal cycling. The MVC test consisted of five isometric MVC trials with right elbow flexion, using a work/rest ratio of $2 / 5 \mathrm{~s}$. The specific warm-up consisted of five 2-s isometric contractions where the exerted force was equal to approximately $50 \%$ of the subjects' perceived maximum force, followed by $5 \mathrm{~s}$ of rest. During the MVC tests, subjects were verbally encouraged to perform maximally, and a visual feedback was provided. After each subject rested for 8 min on the cycle ergometer, they performed the 6-min submaximal cycling. Each subject's feet were strapped to the pedals to prevent them from slipping. The seat height was adjusted so that there was a slight bend in the knee joint when the foot pedal was at its lowest position. During the submaximal cycling, the subjects were constrained to maintain a pedaling frequency of $60 \mathrm{rpm}$ against the prescribed workload (LI: $38.0 \% \pm 4.1 \% \mathrm{~W}_{\max }$ : $72 \pm 14 \mathrm{~W}$; MI: $66.6 \% \pm 2.0 \% \mathrm{~W}_{\max }$ : $125 \pm 14 \mathrm{~W}$; HI: $\left.78.4 \% \pm 5.2 \% \mathrm{~W}_{\max }: 147 \pm 18 \mathrm{~W}\right)$. During the submaximal cycling, subjects were reminded to relax the right biceps brachii (BB) muscle as much as possible and the experimenter monitored the muscle activity of bilateral BB muscles using electromyogram (EMG). The MVC tests were also performed immediately after (POST-0), 5 min (POST-5), and $10 \mathrm{~min}$ (POST-10) after the submaximal cycling. 
The isometric force exerted by the right elbow flexion was measured with the shoulder at $90^{\circ}$ abduction and the elbow at $90^{\circ}$ flexion. The right upper arm was strapped to a fixed platform for support. The wrist was inserted into a padded non-compliant strap attached to a strain-gauge transducer (T.K.K.1269f, Takei Scientific Instruments Co., Ltd., Niigata, Japan). The measured force signals were fed into a digital dynamometer (T.K.K.1269, Takei Scientific Instruments Co., Ltd.) to amplify them and display the force values in digits. A surface EMG was recorded using bipolar EMG sensors (interelectrode distance of $10 \mathrm{~mm}$; DE-2.1, Delsys, Inc., Natick, MA) positioned over the muscle belly of the right BB muscle. Before attachment of the sensors, the skin was shaved, abraded, and cleaned with alcohol to reduce skin impedance. The ground electrode was placed over the left spine of the scapula. The position of the sensors was marked on the skin to ensure constant positioning of the sensors throughout all sessions. EMG signals were amplified (Bagnoli-4 System, Delsys, Inc.) with band-pass filtering between 20 and $450 \mathrm{~Hz}$. The amplified force and EMG signals were stored in a computer with a sampling rate of $1 \mathrm{kHz}$ using an analog-digital converter (PowerLab 8/30, ADInstruments, Sydney, Australia) for offline analysis later (LabChart v8.1.3 for Windows, ADInstruments). The mean force and root mean square (RMS) of EMG for each MVC trial were calculated from a 0.1-s window before the peak force of each contraction, and these values were defined as peak force and peak RMS, respectively. Furthermore, the RMS of EMG of right BB muscle for the submaximal cycling was calculated from a 1-min window for every $1 \mathrm{~min}$. These values were then normalized to the highest value in the PRE and reported as a percentage (9).

Blood sampling was performed from the fingertips using capillary tubes at rest ( $3 \mathrm{~min}$ before the initiation of submaximal cycling: PRE), at 3 min during the cycling (CYC), and immediately before the POST-0, POST-5, and POST-10 MVC tests. Samples of $25 \mu 1$ were analyzed using a lactate analyzer (YSI 1500 SPORT, YSI, Yellow Springs, OH) to measure the lactate concentration ([La]). The lactate analyzer was calibrated by a standard lactate solution of $5 \mathrm{mmol} / \mathrm{L}$ before each session.

The $\dot{\mathrm{VO}}_{2}, \dot{\mathrm{V} C O}{ }_{2}$, ventilation ( $\dot{\mathrm{VE}}$ ), and heart rate (HR) were measured continuously, using a respiratory gas analyzer (AE-300S, Minato Medical Science, Osaka, Japan) during rest, exercise, and recovery periods. For each 15-s interval, the average of each cardiorespiratory variable was calculated. Inspired and expired flows were measured using a hot-wire flow meter that was linear with respect to a flow range of 0-600 L/min. A zirconium sensor and infrared absorption analyzer were used to analyze the inspired and expired fractions of $\mathrm{O}_{2}$ and $\mathrm{CO}_{2}$, respectively. The flow meter and gas analyzers were calibrated prior to each session with a standard 2- $\mathrm{L}$ syringe and precision reference gas $\left(\mathrm{O}_{2} 15.0 \%\right.$ and $\mathrm{CO}_{2}$ 5.0\%). Data for $\dot{\mathrm{VO}_{2}}, \dot{\mathrm{VE}}$, and HR were obtained at CYC and immediately before PRE, POST-0, POST-5, and POST-10 MVC tests.

For the normalized peak force and peak RMS, three-way analysis of variance (ANOVA) with repeated measures was performed with MVC trial, time, and condition as factors. For RMS during a submaximal cycling, [La], and cardiorespiratory responses, a two-way ANOVA with repeated measures was performed with time and condition as factors. All variables were examined using Mendoza's multisample sphericity test. Whenever the data violated the assumption of sphericity, $P$ values based on the Greenhouse-Geisser correction were reported. After ANOVA, Shaffer's modified sequentially rejective Bonferroni procedure was performed for multiple comparisons. Partial $\eta$-squared $\left(\eta_{p}^{2}\right)$ and Cohen's $d$ values are presented as measures of effect size. For absolute peak force and peak RMS, intraclass correlation coefficient (ICC) was calculated to evaluate the reproducibility of the highest 
value in the PRE among the three conditions. $P$ value of $<0.05$ was regarded as statistically significant. Values of the results are given as mean \pm SEM.

\section{Results}

The $\mathrm{ICC}_{(1,1)}$ of the PRE tests for absolute peak force and peak RMS across the three conditions are as follows: absolute peak force $(r=0.79)$ and peak RMS $(r=0.90)$. These variables showed substantial or almost perfect reliability.

The normalized peak force of right elbow flexion was not impaired after the submaximal cycling $\left[F_{(3,21)}=1.21, P=0.33, \eta_{p}^{2}=0.15\right]$ and was not significantly different across the conditions $\left[F_{(2,14)}=0.09, P=0.92, \eta_{p}^{2}=0.01\right.$; Fig. 2]. No significant three-way $\left[F_{(24,168)}=\right.$ $1.17, P=0.28, \eta_{p}^{2}=0.14$ ] or two-way interactions [condition and time, $F_{(3.34,23.39)}=0.56$, $\varepsilon=0.48, P=0.67, \eta_{p}^{2}=0.07$; condition and MVC trial, $F_{(8,56)}=1.48, P=0.19, \eta_{p}^{2}=0.17$; time and MVC trial, $\left.F_{(12,84)}=0.94, P=0.51, \eta_{p}^{2}=0.12\right]$ were observed for the MVC force. A significant main effect was found for $\operatorname{MVC}$ trial $\left[F_{(4,28)}=4.05, P=0.01, \eta_{p}^{2}=0.37\right]$, and post hoc tests showed that the peak force was reduced in the fourth trial as compared with the second trial in all of the time points $(P=0.04, d=0.41)$.

Normalized peak RMS in the right BB muscle was not influenced by the submaximal cycling $\left[F_{(1.45,10.12)}=1.81, \varepsilon=0.48, P=0.21, \eta_{p}^{2}=0.21\right]$ and was not significantly different across the conditions $\left[F_{(2,14)}=1.09, P=0.36, \eta_{p}^{2}=0.13\right.$; Fig. 3]. No significant three-way $\left[F_{(24,168)}=0.72, P=0.83, \eta_{p}^{2}=0.09\right]$ or two-way interactions [condition and time, $F_{(2.19,15.34)}=1.81, \varepsilon=0.36, P=0.20, \eta_{p}^{2}=0.20 ;$ condition and MVC trial, $F_{(3.87,27.07)}=0.60, \varepsilon=0.48, P=0.66, \eta_{p}^{2}=0.08$; time and MVC trial, $F_{(12,84)}=0.91$, $\left.P=0.54, \eta_{p}^{2}=0.12\right]$ were observed for the peak RMS. A significant main effect was found for MVC trial $\left[F_{(4,28)}=2.90, P=0.04, \eta_{p}^{2}=0.29\right]$, but post hoc tests did not show any significant differences across the MVC trials. During the cycling session, changes in RMS were not significantly different between the conditions [interaction, $F_{(2.12,14.87)}=2.26$, $\varepsilon=0.21, P=0.14, \eta_{p}^{2}=0.24$; main intensity, $F_{(2,14)}=2.43, P=0.12, \eta_{p}^{2}=0.26$; main time, $\left.F_{(5,35)}=6.11, P<0.001, \eta^{2}=0.47\right]$. Post hoc tests did not reveal any significant differences across the time points.

[La] had significantly increased after submaximal cycling, and the rate of increase was significantly different between the conditions [interaction, $F_{(2.9,20.28)}=41.83, \varepsilon=0.36$,
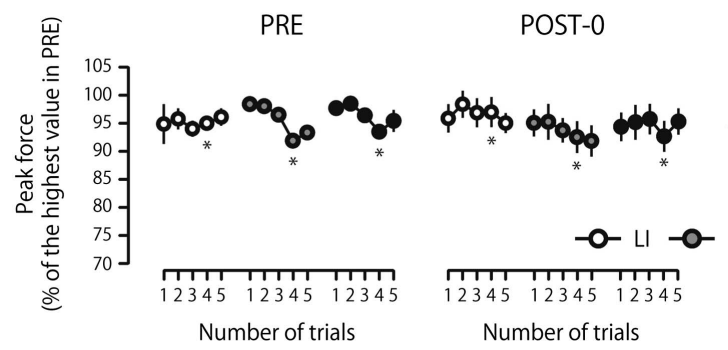

POST-5

POST-10

Fig. 2. Peak force during multiple maximal voluntary contractions (MVCs) tests performed before (PRE), immediately after (POST-0), 5 min after (POST-5), and 10 min after (POST-10) a 6-min submaximal cycling task at low (LI, open circles), moderate (MI, gray circles), or high intensities (HI, filled circles). *Significant difference versus 2 nd trial in iso-tests $(P<0.05)$ 


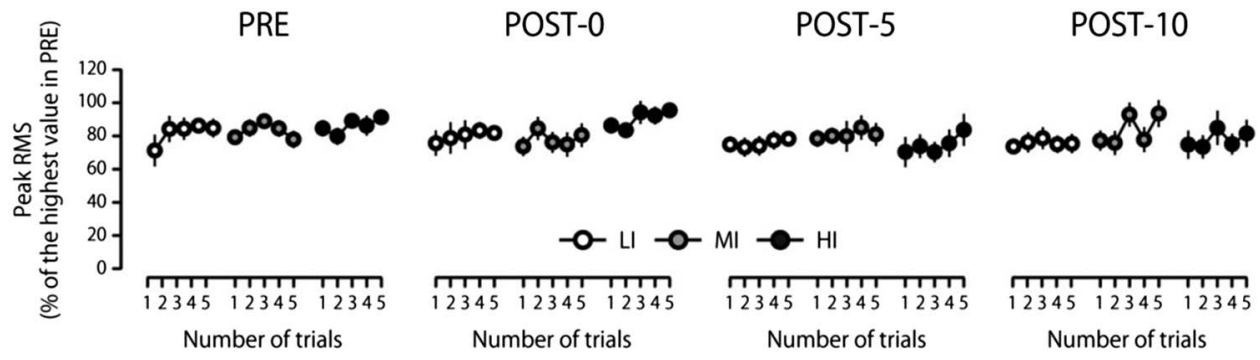

Fig. 3. Peak root mean square (RMS) of electromyogram recorded from the right biceps brachii muscle during multiple maximal voluntary contractions (MVCs) tests performed before (PRE), immediately after (POST-0), 5 min after (POST-5), and 10 min after (POST-10) a 6-min submaximal cycling task at low (LI, open circles), moderate (MI, gray circles), or high intensities (HI, filled circles)

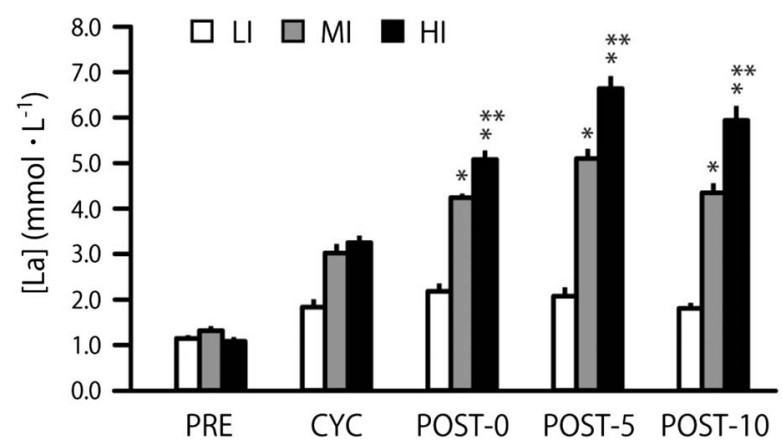

Fig. 4. Blood lactate concentration ([La]) at rest (PRE), 3 min during (CYC), immediately after (POST-0), 5 min after (POST-5), and 10 min after (POST-10) the 6-min submaximal cycling at low (LI, open bars), moderate (MI, gray bars), or high intensities (HI, filled bars).

* Significant difference versus LI at isotime $(P<0.001)$. **Significant difference versus $\mathrm{MI}$ at isotime $(P<0.01)$

$P<0.001, \quad \eta_{p}^{2}=0.86 ;$ LI, $\left.F_{(2.16,} 15.11\right)=19.67, \varepsilon=0.54, \quad P<0.001, \quad \eta_{p}^{2}=0.74 ; \quad$ MI, $F_{(1.54,10.78)}=81.30, \varepsilon=0.38, P<0.001, \eta_{p}^{2}=0.92$; HI, $F_{(1.59,11.15)}=132.49, \varepsilon=0.40$, $P<0.001, \eta_{p}^{2}=0.95$; Fig. 4]. [La] was significantly higher in the HI condition than in the LI and MI conditions and was significantly higher in the MI condition than in the LI condition at POST-0 $\left[F_{(2,14)}=131.99, P<0.001, \eta_{p}^{2}=0.95\right.$; LI vs. MI, $P<0.001, d=5.49$; LI vs. HI, $P<0.001, d=5.58 ;$ MI vs. HI, $P<0.001, d=1.93]$, POST $-5\left[F_{(2,14)}=151.52\right.$, $P<0.001, \eta_{p}^{2}=0.96$; LI vs. MI, $P<0.001, d=5.28$; LI vs. HI, $P<0.001, d=6.83$; MI vs. HI, $P<0.001, d=2.24]$, and POST-10 time points $\left[F_{(2,14)}=97.14, P<0.001\right.$, $\eta_{p}^{2}=0.93$; LI vs. MI, $P<0.001, d=5.42$; LI vs. HI, $P<0.001, d=6.09$; MI vs. HI, $P<0.001, d=2.09]$.

The cardiorespiratory responses of the participants are summarized in Table $\mathrm{I}$. $\mathrm{VO}_{2}$ significantly increased after submaximal cycling, and the rate of increase was significantly different between the conditions [interaction, $F_{(8,56)}=80.96, P<0.001, \eta_{p}^{2}=0.92$; LI, $F_{(2.11,14.77)}=152.87, \varepsilon=0.53, P<0.001, \eta_{p}^{2}=0.96$; MI, $F_{(1.51,10.59)}=392.73, \varepsilon=0.38$, $\left.P<0.001, \eta^{2}=0.98 ; \mathrm{HI}, F_{(1.38,9.65)}=401.84, \varepsilon=0.34, P<0.001, \eta^{2}{ }_{p}=0.98\right]$. At POST-0, $\mathrm{VO}_{2}$ was significantly higher in the $\mathrm{HI}$ condition than in the LI and MI conditions and was significantly higher in the MI condition than in the LI condition $\left[F_{(1.21,8.5)}=289.55\right.$, $\varepsilon=0.61, P<0.001, \eta_{p}^{2}=0.98$; LI vs. MI, $P<0.001, d=3.41$; LI vs. HI, $P<0.001, d=$ 4.68; MI vs. HI, $P<0.001, d=1.51]$. $\dot{\mathrm{VO}}_{2}$ was not significantly different between the conditions at POST $-5\left[F_{(2,14)}=2.98, P=0.08, \eta_{p}^{2}=0.30\right]$ and POST-10 time points $\left[F_{(2,14)}=0.66\right.$, $\left.P=0.53, \eta_{p}^{2}=0.09\right]$. VE significantly increased after submaximal cycling, and the rate of 
Table I. Cardiorespiratory responses during and after the 6-min submaximal cycling task

\begin{tabular}{|l|c|c|c|c|c|c|}
\hline & & PRE & CYC & POST-0 & POST-5 & POST-10 \\
\hline \multirow{2}{*}{$\dot{V O}_{2}(\mathrm{ml} / \mathrm{min})$} & $\mathrm{LI}$ & $381(21)$ & $1248(71)$ & $1237(68)$ & $416(43)$ & $418(46)$ \\
\cline { 2 - 7 } & $\mathrm{MI}$ & $366(43)$ & $1895(71)$ & $1921(74)^{*}$ & $480(26)$ & $459(38)$ \\
\cline { 2 - 7 } & $\mathrm{HI}$ & $363(24)$ & $2055(79)$ & $2263(86)^{* * * *}$ & $481(18)$ & $458(24)$ \\
\hline \multirow{2}{*}{$\dot{\mathrm{VE}}(\mathrm{L} / \mathrm{min})$} & $\mathrm{LI}$ & $13.8(0.4)$ & $35.9(2.6)$ & $36.9(2.2)$ & $16.1(1.2)$ & $16.7(2.1)$ \\
\cline { 2 - 7 } & $\mathrm{MI}$ & $13.6(1.3)$ & $58.0(2.6)$ & $57.5(2.2)^{*}$ & $20.2(1.0)^{*}$ & $17.8(1.6)$ \\
\cline { 2 - 7 } & $\mathrm{HI}$ & $13.6(0.9)$ & $67.3(2.7)$ & $80.8(3.5)^{* * * *}$ & $23.4(1.1)^{* * * *}$ & $19.7(1.0)$ \\
\hline \multirow{2}{*}{$\mathrm{HR}(\mathrm{bpm})$} & $\mathrm{LI}$ & $84(3)$ & $110(2)$ & $115(2)$ & $92(3)$ & $87(3)$ \\
\cline { 2 - 7 } & MI & $86(3)$ & $138(6)$ & $148(6)^{*}$ & $103(5)^{*}$ & $98(4)^{*}$ \\
\cline { 2 - 7 } & HI & $83(3)$ & $150(4)$ & $163(6)^{*}$ & $113(4)^{*}$ & $103(3)^{*}$ \\
\hline
\end{tabular}

LI: low-intensity condition; MI: moderate-intensity condition; HI: high-intensity condition; PRE: time point before the cycling; CYC: time point at 3 min during the cycling; POST-0: time point immediately after the cycling; POST-5: time point $5 \mathrm{~min}$ after the cycling; POST-10: time point $10 \mathrm{~min}$ after the cycling, $\mathrm{VO}_{2}$ : oxygen uptake; VE: ventilation; $\mathrm{HR}$, heart rate.

* Significant difference versus LI $(P<0.05)$.

${ }^{* *}$ Significant difference versus MI $(P<0.05)$

increase was significantly different between the conditions [interaction, $F_{(3,20.98)}=61.32$, $\varepsilon=0.37, P<0.001, \eta_{p}^{2}=0.90 ;$ LI, $F_{(1.93,13.52)}=75.10, \varepsilon=0.48, P<0.001, \quad \eta_{p}^{2}=0.91$; MI, $F_{(4,28)}=205.07, P<0.001, \eta_{p}^{2}=0.97 ; \mathrm{HI}, F_{(1.3,9.09)}=237.81, \varepsilon=0.32, P<0.001$, $\left.\eta_{p}^{2}=0.97\right]$. VE was significantly higher in the HI condition than in the LI and MI conditions and was significantly higher in the MI condition than in the LI condition at POST- $0\left[F_{(1.22,8.56)}=\right.$ 122.44, $\varepsilon=0.61, P<0.001, \eta_{p}^{2}=0.94$; LI vs. MI, $P<0.001, d=1.86$; LI vs. HI, $P<0.001$, $d=2.18$; MI vs. HI, $P<0.001, d=0.70]$ and POST-5 time points $\left[F_{(1.1,7.73)}=\right.$ 27.74, $\varepsilon=0.54, P<0.001, \eta_{p}^{2}=0.80$; LI vs. MI, $P<0.001, d=1.28$; LI vs. HI, $P<0.001, d=2.24$; MI vs. HI, $P=0.03, d=1.07]$. At POST-10 time point $\left[F_{(2,14)}=\right.$ $\left.1.86, P=0.19, \eta_{p}^{2}=0.21\right]$, VE was not significantly different between the conditions. The rate of increase in $\mathrm{HR}$ was significantly different between the conditions [interaction, $F_{(3.11,21.76)}=18.35, \varepsilon=0.39, P<0.001, \eta_{p}^{2}=0.72 ;$ LI, $F_{(1.97,13.77)}=45.51, \varepsilon=0.49$, $P<0.001, \quad \eta_{p}^{2}=0.87 ;$ MI, $F_{(1.81,12.67)}=85.31, \varepsilon=0.45, P<0.001, \quad \eta_{p}^{2}=0.92 ; \quad \mathrm{HI}$, $\left.F_{(4,28)}=114.77, P<0.001, \eta_{p}^{2}=0.94\right]$. HR was significantly higher in the HI and MI conditions than in the LI condition at POST-0 $\left[F_{(2,14)}=32.29, P<0.001, \eta^{2}=0.82\right.$; LI vs. MI, $P<0.01, d=2.54$; LI vs. HI, $P<0.001, d=3.80]$, POST-5 $\left[F_{(2,14)}=7.64, P<0.01\right.$, $\eta_{p}^{2}=0.52$; LI vs. MI, $P=0.02, d=0.99$; LI vs. HI, $\left.P=0.02, d=2.05\right]$, and POST-10 time points $\left[F_{(2,14)}=27.60, P<0.001, \eta_{p}^{2}=0.80\right.$; LI vs. MI, $P<0.001, d=1.05$; LI vs. HI, $P<0.01, d=1.75]$.

\section{Discussion}

Contrary to our hypothesis, MVC force of elbow flexion after cycling was not different across the three intensity conditions. These results indicate that different changes in the metabolic 
milieu of the exercised muscle induced by voluntary exercise may not influence MVC force of elbow flexor muscles. To our knowledge, this is the first study to show that different changes in metabolic milieu induced by submaximal cycling do not influence force during intermittent MVC of elbow flexion, without using pharmacological technique or circulatory occlusion.

This study was based on the premise that an increase in exercise intensity of the submaximal cycling would be accompanied by an enhancement in group III/IV muscle afferent feedback to the central nervous system. Direct assessment of the afferent feedback is currently not possible (3). Although the direct assessment could also not be performed in this study, several results of this study indicate that the premise was likely to be valid. First, the more intense the exercise intensity of the 6-min submaximal cycling was, the higher the [La] was after the cycling. Group III/IV muscle afferents were demonstrated to respond to the levels of lactate, ATP, and protons $(2,18)$. It is likely that the difference in [La] between the conditions supports the premise. Second, group III/IV muscle afferent feedback is also related to a regulation of cardiorespiratory responses to exercise (22). VE and HR were significantly different between the conditions after the cycling. These results suggest that the three intensity conditions resulted in different changes in group III/IV muscle afferent feedback after the cycling. Cardiorespiratory responses are also regulated by central motor command $(15,16,26)$. However, it is likely that the differences in cardiorespiratory responses resulted from the differences in group III/IV muscle afferent feedback since cardiorespiratory responses differed in the absence of central motor command (i.e., the POST-5 time point). Altogether, the current results indicate that group III/IV muscle afferent feedback from the exercised leg enhanced in proportion to an increase in exercise intensity of the submaximal cycling, until $5 \mathrm{~min}$ after the cycling. This interpretation for group III/IV afferent feedback is similar to that in the study by Amann et al. (2).

As stated above, the 6-min submaximal cycling did not reduce MVC force of elbow flexion across the three conditions. Considering the current results that support the hypothesis that group III/IV muscle afferent feedback was different across the conditions, it is likely that changes in group III/IV muscle afferent feedback induced by the 6-min submaximal cycling did not cause NLMF in the elbow flexor muscles. It has been previously reported that MVC force of single elbow flexion was reduced by only $5 \%$ after a constant-workload cycling of $80 \%$ peak power output to failure (20). Since [La] after the cycling to failure was greater compared with that in this study, it is likely that changes in group III/IV muscle afferent feedback were greater compared with those resulted from the cycling in this study. This suggests that slight NLMF effects necessitate extensive changes in group III/IV muscle afferent feedback induced by fatiguing exercise. In the previous studies showing NLMF effects of lower-limb dynamic exercise on upper limb muscles, the fatiguing task included cycling in hypoxic conditions $(17)$ or until exhaustion $(17,20)$ and resulted in higher [La] $\left(>8 \mathrm{mmol} \mathrm{L}^{-1}\right.$ ) at the end of the exercise than that in the HI condition. These results support the idea that the severe metabolic milieu induced by dynamic exercise may cause NLMF. Additionally, the duration of fatiguing task was longer in the previous studies ( $>9 \mathrm{~min}$ ) than in this study (6 min). For long-duration submaximal contractions or whole body exercise, greater supraspinal fatigue occurs $(7,23,24)$. Since NLMF has been attributed to central mechanisms $(1,8,9,14)$, a shorter duration of fatiguing task may also be related to no NLMF effects in this study. In a pilot study, several subjects of this study could not maintain the power output at $80 \%$ of $\mathrm{VO}_{2 \text { peak }}$ for over $6 \mathrm{~min}$, resulting in the choice of $6 \mathrm{~min}$ as the duration of cycling. This might be due to the subjects' arms being relaxed in the air (i.e., not 
gripping the ergometer handle). Indeed, the absolute value of work rate at $\dot{\mathrm{VO}}_{2 \text { peak }}$ and the values of HR at the end of submaximal cycling were low in this study. An experimental setting in which both arms remain relaxed is important to prevent confounding effects of activation of arm muscles in the study for NLMF in the non-exercised arm. As the absolute value of work rate at $\mathrm{VO}_{2 \text { peak }}$ is higher in cycling in the spine position (4), in which both arms remain inactive, than that in this study, body posture should be considered in further research. These low $\dot{\mathrm{VO}}_{2 \text { peak }}$ and HR values suggest that subjects terminated the cycling exercise before cardiorespiratory responses would become maximal. Since the exercise intensity of the submaximal cycling was determined relative to $\mathrm{VO}_{2 \text { peak }}$ achieved by the incremental cycling with both arms relaxed, the variety of physiological stress undergone during the only leg cycling in this study might be less than that during whole body exercise (i.e., traditional leg cycling). However, it is likely that changes in the metabolic milieu induced by 6 min of submaximal cycling at $40 \%-80 \%$ of $\mathrm{W}_{\max }$ exerted by leg cycling without body support do not determine the magnitude of NLMF effects on the non-exercised upper limb muscles.

It has been argued that, in the non-exercised muscle, force loss during multiple MVCs protocol is greater than that during a single-MVC protocol after fatiguing contractions (8). Several studies have shown no NLMF effects with a single MVC $(3,8,25)$. In contrast, NLMF effects were observed in the last five MVCs of the intermittent 12 MVCs (8). Hence, this study applied the multiple MVCs protocol as a test to investigate NLMF effects. However, the number of MVCs during the multiple MVCs protocol was less in this study than in the previous study showing NLMF effects (8) in order to minimalize the effects of fatigue resulting from multiple MVCs on subsequent MVC tests. If a greater number of MVCs were performed in this study, MVC force might be reduced in the latter contractions during MVCs test after the cycling. However, further studies are needed to elucidate the relationship between NLMF effects and testing protocols for non-exercised muscles.

During the submaximal cycling, the RMS in the right BB muscle increased with time. These results suggest that complete relaxation was not achieved in either of elbow flexor muscle. It is possible that this muscle activity influenced MVC force after the cycling. However, the activity was not different across the three intensity conditions. Therefore, the effects of activation of the BB muscles on MVC force and EMG after the cycling can be ruled out in this study.

In this study, forearm and biceps muscle temperatures were not measured. An increase in muscle temperature after an active warm-up improved peak power output during squat jump (21). However, an increase in muscle temperature after an active warm-up did not change maximal force output during isometric contraction (21). Therefore, it can be inferred that the difference in forearm and biceps muscle temperature across the conditions did not influence MVC force of elbow flexion after the submaximal cycling in this study.

In conclusion, changes in the metabolic milieu induced by 6 min of submaximal cycling at $40 \%-80 \%$ of $\mathrm{W}_{\max }$, exerted by cycling without body support, do not determine the magnitude of the NLMF effects on the non-exercised upper limb muscles. This finding suggests that changes in group III/IV muscle afferent feedback induced by 6 min of submaximal cycling do not cause NLMF effects. The results from this study may be helpful in the development of new exercise training or warm-up methods.

\section{Acknowledgements}

This study was supported by a Grant-in-Aid for Scientific Research from the Japanese Ministry of Education, Science, and Culture (JSPS KAKENHI Grant Number 21800083). 


\section{REFERENCES}

1. Aboodarda SJ, Copithorne DB, Power KE, Drinkwater E, Behm DG: Elbow flexor fatigue modulates central excitability of the knee extensors. Appl. Physiol. Nutr. Metab. 40, 924-930 (2015)

2. Amann M, Sidhu SK, Weavil JC, Mangum TS, Venturelli M: Autonomic responses to exercise: group III/IV muscle afferents. Auton. Neurosci. 188, 19-23 (2015)

3. Amann M, Venturelli M, Ives SJ, McDaniel J, Layec G, Rossman MJ, Richardson RS: Peripheral fatigue limits endurance exercise via a sensory feedback-mediated reduction in spinal motoneuronal output. J. Appl. Physiol. 115, 355-364 (2013)

4. Arimitsu T, Matsuura R, Yunoki T, Yamanaka R, Kimura T, Lian CS, Afroundeh R, Yano T: Relationship between oxygen uptake and oxygen supply system during incremental-load supine exercise. Biol. Sport 28, 83-87 (2011)

5. Beaver WL, Wasserman K, Whipp BJ: A new method for detecting anaerobic threshold by gas exchange. J. Appl. Physiol. 60, 2020-2027 (1986)

6. Birdsong WT, Fierro L, Williams FG, Spelta V, Naves LA, Knowles M, Marsh-Haffner J, Adelman JP, Almers W, Elde RP, McCleskey EW: Sensing muscle ischemia: coincident detection of acid and ATP via interplay of two ion channels. Neuron 68, 739-749 (2010)

7. Gandevia SC, Allen GM, Butler JE, Taylor JL: Supraspinal factors in human muscle fatigue: evidence for suboptimal output from the motor cortex. J. Physiol. 490, 529-536 (1996)

8. Halperin I, Aboodarda SJ, Behm DG: Knee extension fatigue attenuates repeated force production of the elbow flexors. Eur. J. Sport Sci. 14, 823-829 (2014)

9. Halperin I, Copithorne D, Behm DG: Unilateral isometric muscle fatigue decreases force production and activation of contralateral knee extensors but not elbow flexor. Appl. Physiol. Nutr. Metab. 39, 1338-1344 (2014)

10. Jankowski MP, Rau KK, Ekmann KM, Anderson CE, Koerber HR: Comprehensive phenotyping of group III and IV muscle afferents in mouse. J. Neurophysiol. 109, 2374-2381 (2013)

11. Johnson MA, Sharpe GR, Williams NC, Hannah R: Locomotor muscle fatigue is not critically regulated after prior upper body exercise. J. Appl. Physiol. 119, 840-850 (2015)

12. Kennedy DS, Fitzpatrick SC, Gandevia SC, Taylor JL: Fatigue-related firing of muscle nociceptors reduces voluntary activation of ipsilateral but not contralateral lower limb muscles. J. Appl. Physiol. 118, 408-418 (2015)

13. Kennedy DS, McNeil CJ, Gandevia SC, Taylor JL: Fatigue-related firing of distal muscle nociceptors reduces voluntary activation of proximal muscles of the same limb. J. Appl. Physiol. 116, 385-394 (2014)

14. Kennedy DS, McNeil CJ, Gandevia SC, Taylor JL: Firing of antagonist small-diameter muscle afferents reduces voluntary activation and torque of elbow flexors. J. Physiol. 591, 3591-3604 (2013)

15. Marcora SM, Bosio A, de Morree HM: Locomotor muscle fatigue increases cardiorespiratory responses and reduces performance during intense cycling exercise independently from metabolic stress. Am. J. Physiol. Regul. Integr. Comp. Physiol. 294, R874-R883 (2008)

16. Nicolo A, Marcora SM, Sacchetti M: Respiratory frequency is strongly associated with perceived exertion during time trials of different duration. J. Sports Sci. 34, 1199-1206 (2016)

17. Rasmussen P, Nielsen J, Overgaard M, Krogh-Madsen R, Gjedde A, Secher NH, Petersen NC: Reduced muscle activation during exercise related to brain oxygenation and metabolism in humans. J. Physiol. 588, 1985-1995 (2010)

18. Rotto DM, Kaufman MP: Effect of metabolic products of muscular contraction on discharge on group III and IV afferents. J. Appl. Physiol. 64, 2306-2313 (1988)

19. Šambaher N, Aboodarda SJ, Behm DG: Bilateral knee extensor fatigue modulates force and responsiveness of the corticospinal pathway in the non-fatigued, dominant elbow flexors. Front. Hum. Neurosci. 10, 18 (2016)

20. Sidhu SK, Weavil JC, Venturelli M, Garten RS, Rossman MJ, Richardson RS, Gmelch BS, Morgan DE, Amann M: Spinal $\mu$-opioid receptor-sensitive lower limb muscle afferents determine corticospinal responsiveness and promote central fatigue in upper limb muscle. J. Physiol. 592, 5011-5024 (2014)

21. Stewart D, Macaluso A, De Vito G: The effect of an active warm-up on surface EMG and muscle performance in healthy humans. Eur. J. Appl. Physiol. 89, 509-513 (2003)

22. Taylor JL, Amann M, Duchateau J: Neural contributions to muscle fatigue: from the brain to the muscle and back again. Med. Sci. Sports Exerc. 48, 2294-2306 (2016)

23. Taylor JL, Gandevia SC: A comparison of central aspects of fatigue in submaximal and maximal voluntary contraction. J. Appl. Physiol. 104, 542-550 (2008) 
24. Thomas K, Goodall S, Stone M, Howatson G, Gibson AS, Ansley L: Central and peripheral fatigue in male cyclists after 4-, 20-, and 40-km time trials. Med. Sci. Sports Exerc. 47, 537-546 (2015)

25. Triscott S, Gordon J, Kuppuswamy A, King N, Davey N, Ellaway P: Differential effects of endurance and resistance training on central fatigue. J. Sports Sci. 26, 941-951 (2008)

26. Williamson JW: The relevance of central command for the neural cardiovascular control of exercise. Exp. Physiol. 95, 1043-1048 (2010) 